\title{
Orai1 contributes to the establishment of an apoptosis-resistant phenotype in prostate cancer cells
}

\author{
M Flourakis ${ }^{1,2,6,7}$, V Lehen'kyi ${ }^{1,2,6}$, B Beck ${ }^{1,2,6}, M$ Raphaël ${ }^{1,2}, M$ Vandenberghe ${ }^{1,2}$, FV Abeele ${ }^{1,2}$, M Roudbaraki $^{1,2}$, G Lepage $^{1,2}$, \\ B Mauroy $^{3}$, C Romanin ${ }^{4}$, Y Shuba ${ }^{5}$, R Skryma ${ }^{1,2}$ and N Prevarskaya ${ }^{\star, 1,2}$
}

The molecular nature of calcium $\left(\mathrm{Ca}^{2+}\right)$-dependent mechanisms and the ion channels having a major role in the apoptosis of cancer cells remain a subject of debate. Here, we show that the recently identified Orai1 protein represents the major molecular component of endogenous store-operated $\mathrm{Ca}^{2+}$ entry (SOCE) in human prostate cancer (PCa) cells, and constitutes the principal source of $\mathrm{Ca}^{2+}$ influx used by the cell to trigger apoptosis. The downregulation of Orai1, and consequently SOCE, protects the cells from diverse apoptosis-inducing pathways, such as those induced by thapsigargin ( $\mathrm{Tg})$, tumor necrosis factor $\alpha$, and cisplatin/oxaliplatin. The transfection of functional Orai1 mutants, such as R91W, a selectivity mutant, and L273S, a coiled-coil mutant, into the cells significantly decreased both SOCE and the rate of Tg-induced apoptosis. This suggests that the functional coupling of STIM1 to Orai1, as well as Orai1 $\mathrm{Ca}^{2+}$-selectivity as a channel, is required for its pro-apoptotic effects. We have also shown that the apoptosis resistance of androgen-independent PCa cells is associated with the downregulation of Orai1 expression as well as SOCE. Orai1 rescue, following Orai1 transfection of steroid-deprived cells, re-established the storeoperated channel current and restored the normal rate of apoptosis. Thus, Orai1 has a pivotal role in the triggering of apoptosis, irrespective of apoptosis-inducing stimuli, and in the establishment of an apoptosis-resistant phenotype in PCa cells.

Cell Death and Disease (2010) 1, e75; doi:10.1038/cddis.2010.52; published online 16 September 2010

Subject Category: Cancer

Early and pivotal events in apoptosis are now known to occur in mitochondria and the endoplasmic reticulum (ER), where the release of cytochrome $c$ from the mitochondria and calcium $\left(\mathrm{Ca}^{2+}\right)$ from the ER into the cytosol is a requisite for apoptosis in many cases. ${ }^{1}$ Irrespective of apoptosis-induced stimuli, a lethal influx of $\mathrm{Ca}^{2+}$ constitutes a sine qua non condition of apoptosis. The recruitment of three major $\mathrm{Ca}^{2+}$ dependent apoptotic mechanisms, mitochondrial, cytoplasmic and ER, were already shown (for reviews, see Prevarskaya et al., ${ }^{2}$ Pinton et al. ${ }^{3}$ and Norberg et al. ${ }^{4}$ ). However, until now, the specific mechanisms through which $\mathrm{Ca}^{2+}$ dynamics are controlled and by which $\mathrm{Ca}^{2+}$ participates in apoptotic cascades have been elusive. The function of $\mathrm{Ca}^{2+}$ in apoptosis is particularly fascinating, especially when we consider the prominence of $\mathrm{Ca}^{2+}$ in regulating a multitude of physiological processes and the involvement of perturbed cellular $\mathrm{Ca}^{2+}$ homeostasis in the pathogenesis.

Prostate cancer $(\mathrm{PCa})$ is the second most lethal tumor among men, wherein the major hallmark is the acquired resistance to apoptosis rather than enhanced proliferation. .,6 $^{5}$ The early stage of PCa depends on the androgens needed for growth and survival, and androgen ablation therapy may at this time be effective in causing the tumor to regress due to the induction of massive apoptosis. ${ }^{7}$ Unfortunately, PCa progresses into an androgen-independent stage, causing cancer relapse with the appearance of more aggressive cell phenotypes characterized by enhanced apoptosis resistance. Despite a growing number of studies, the mechanisms leading to these phenotypes are still poorly defined, even though understanding the factors that drive $\mathrm{PCa}$ to apoptosis resistance is vital for the development of new therapies for advanced $\mathrm{PCa}$.

A number of studies have shown that a large, sustained influx of $\mathrm{Ca}^{2+}$ triggering apoptosis in cancer cells is provided by capacitative or store-operated $\mathrm{Ca}^{2+}$ entry (capacitative calcium entry (CCE) or store-operated calcium entry (SOCE)), mediated by store-operated channels (SOCs). ${ }^{8,9}$ SOCs are located in the plasma membrane (PM) and are activated by store depletion in the ER. $\mathrm{Ca}^{2+}$ entry via SOCs induces a sustained increase in the cytosolic $\mathrm{Ca}^{2+}$ concentration, thus restoring the $\mathrm{ER} \mathrm{Ca}^{2+}$ content. Therefore, when SOCs are activated, they regulate both cytosolic and

${ }^{1}$ INSERM U1003, Equipe labellisée par la Ligue Nationale contre le cancer, Villeneuve d'Ascq F-59655, France; ${ }^{2}$ Université des Sciences et Technologies de Lille (USTL), Villeneuve d'Ascq F-59655, France; ${ }^{3}$ Université Catholique de Lille, Service d'Urologie, Lille F-59000, France; ${ }^{4}$ Institute for Biophysics, Johannes Kepler Universität Linz, Linz A-4040, Austria and ${ }^{5}$ Bogomoletz Institute of Physiology and International Center of Molecular Physiology NASU, Kyiv 01024, Ukraine

${ }^{*}$ Corresponding author: N Prevarskaya, Laboratoire de Physiologie Cellulaire, INSERM U1003, Equipe labellisée par la Ligue Nationale contre le cancer, Bâtiment SN3, USTL, Villeneuve d'Ascq F-59655, France. Tel: + 3332043 4077; Fax: + 3332043 4066; E-mail: Natacha.Prevarskaya@ univ-lille1.fr

${ }^{6}$ These authors contributed equally to this work.

${ }^{7}$ Current address: Center for Sleep and Circadian Biology, Department of Neurobiology and Physiology, Northwestern University, 2205 Tech Drive, \#2-160, Evanston, IL 60208, USA.

Keywords: store-operated calcium entry; Orai channels; prostate cancer; apoptosis resistance

Abbreviations: AR, androgen receptor; $\mathrm{Ca}^{2+}$, calcium; CCE, capacitative calcium entry; ER, endoplasmic reticulum; ISOC, SOC current; LNCaP, lymph node carcinoma of the prostate cells; LNCaP-ST, steroid-deprived LNCaP; PCa, prostate cancer; PM, plasma membrane; SOCs, store-operated channels; SOCE, store-operated calcium entry; Tg, thapsigargin; TNF $\alpha$, tumor necrosis factor $\alpha$

Received 11.5.10; revised 12.7.10; accepted 02.8.10; Edited by A Finazzi-Agro' 
$\mathrm{ER}$ intraluminal $\mathrm{Ca}^{2+}$ concentrations. This is one reason why SOCs have become of great interest as potential apoptosis regulators. ${ }^{8}$ Moreover, we have previously shown that the inhibition of apoptosis in androgen-independent PCa cells was associated with the downregulation of SOCs due to a decrease in the number of functional channels. ${ }^{9}$ Nevertheless, despite considerable progress in the understanding of SOCs, the molecular nature of the channels involved in $\mathrm{PCa}$ cell apoptosis, and thus contributing to the development of apoptosis resistance, remains unknown.

Our work here focused on studying the molecular mechanisms involved in the enhanced apoptosis resistance of $\mathrm{PCa}$ cells in their transition to the most aggressive hormonerefractory stage.

\section{Results}

Orai1 and STIM1 expression and their involvement in CCE in steroid-deprived PCa cells. The whole-cell configuration of the patch-clamp technique was used to compare the amplitudes of SOC current $\left(I_{S O C}\right)$ in lymph node carcinoma of the prostate cells (LNCaP) and steroiddeprived $\mathrm{LNCaP}$ (LNCaP-ST) cells. As documented in Figure $1 \mathrm{a}-\mathrm{C}, \mathrm{I}_{\mathrm{SOC}}$ induced by the cell dialysis, with the basic intracellular solution supplemented with either $\mathrm{IP}_{3}$ $(100 \mu \mathrm{M})$ or EGTA $(5 \mathrm{mM})($ EGTA + BAPTA), was approximately $50 \%$ lower in LNCaP-ST cells compared with the regular $\mathrm{LNCaP}$ cells. This correlated nicely with the decrease in Orai1 expression in LNCaP-ST cells (Figure 1d) as assayed by the quantitative $\mathrm{PCR}$ and western blotting (Figure 1f). As Orai1 is known to colocalize with the ER $\mathrm{Ca}^{2+}$ sensor, STIM1, at ER-PM junctions and requires STIM1 translocation into punctate structures to be activated, ${ }^{10}$ we also checked for STIM1 expression during steroid deprivation. In contrast to Orai1, the expression of STIM1 remained invariable irrespective of whether or not androgens were present in the culture medium (Figure 1d).

To demonstrate the involvement of STIM1 and Orai1 in Isoc in LNCaP cells, we conducted a series of siRNA-mediated knockdown experiments. As shown in Figure 1e-h, a 24-h treatment with siRNA against Orai1 (si-Orai1) significantly reduced Orai1 mRNA (Figure 1g) and protein (Figure $2 \mathrm{e}$ ) in LNCaP cells. This was paralleled by a decrease in the $\mathrm{IP}_{3}$ - as well as EGTA + BAPTA-evoked $I_{\text {soc }}$ density by $75.4 \pm 7.5 \%$ and $77.8 \pm 11.3 \%$, respectively (Figure 1e). Similarly, the use of siRNA against STIM1 (si-STIM1) specifically decreased STIM1 protein expression by approximately $80 \%$ (Figure 1i) and correlated with a reduction in both $\mathrm{IP}_{3^{-}}$and EGTA + BAPTA-induced $I_{\text {Soc }}$ density by $80.5 \pm 8.4 \%$ and $83.5 \pm 4.2 \%$, respectively (Figure $1 \mathrm{~h}$ ).

\footnotetext{
Downregulation of Orai1 confers apoptosis resistance to LNCaP cells. In view of the major role of Orai1 in PCa cells' SOCE and the decrease in Orai1 expression following androgen withdrawal, we next sought to examine Orai1 involvement in apoptosis. The classic apoptosis inducer, thapsigargin ( $\mathrm{Tg}$, a SERCA pump inhibitor that triggers $\mathrm{Ca}^{2+}$-dependent apoptosis via $\mathrm{ER} \mathrm{Ca}^{2+}$ store depletion and SOCE (e.g., Prevarskaya et al. $\left.{ }^{2}\right)$ ) was used.
}

We used the TUNEL technique (including both negative and positive controls) to measure the apoptosis rate of $\mathrm{LNCaP}$ cells (Figure 2a). Cells were transfected with either si-Orai1 or si-Ctrl and the day after treated with $1 \mu \mathrm{M}$ TG for $24 \mathrm{~h}$. As revealed by TUNEL staining, a 24-h Tg treatment is sufficient to induce apoptosis in around $50 \%$ of $\mathrm{LNCaP}$ cells (Figure $2 \mathrm{~b}$ ). However, knocking down Orai1 expression (with si-Orai1, Figure 2e) made the cells much more resistant to Tg-induced apoptosis ( $4.24 \pm 1.47$ versus $48.4 \pm 4.9 \%$; Figure $2 \mathrm{~b}$ ). This result was confirmed by Hoechst nuclear staining, which revealed $27 \%$ of apoptosis in control cells after a $24-\mathrm{h} \mathrm{Tg}$ treatment and approximately $8 \%$ in si-Orai1-transfected cells (Figure 2c). Thus, Orai1 appears to be an important player in Tg-induced apoptosis, most likely as the key provider of lethal $\mathrm{Ca}^{2+}$ influx in response to $\mathrm{Tg}$-induced $\mathrm{ER} \mathrm{Ca}^{2+}$ store depletion and consecutive SOCE. To validate the role of Orai1 in response to physiological pro-apoptotic signals, we conducted similar experiments with tumor necrosis factor $\alpha$ (TNF $\alpha$ ). TNF $\alpha 10 \mathrm{ng}$ treatment for $48 \mathrm{~h}$ triggered apoptosis in $7.25 \%$ of the control LNCaP cells, and in only $2.5 \%$ of the Orai1-knockdown LNCaP cells (Figure 2d).

To assess whether Orai1 underexpression could be involved in resistance to chemotherapy-induced apoptosis, we also investigated the cisplatin- and oxaliplatin-evoked apoptosis. The use of $20 \mu \mathrm{M}$ cisplatin (Y) and $40 \mu \mathrm{M}$ oxaliplatin $(\mathrm{X})$, two very strong alkylating agents, resulted in an apoptosis rate that was significantly diminished in the Orai1-knockdown LNCaP cells (Figure 3a). Even in the case of these strong alkylating agents si-Orai 1 was able to protect the cells against apoptosis (Figure 3b). Moreover, a 24-h pretreatment with either $20 \mu \mathrm{M}$ cisplatin or $40 \mu \mathrm{M}$ oxaliplatin showed a statistically significant increase in basal $\mathrm{Ca}^{2+}$ level as compared with control DMSO-treated cells (data not shown). These data provide the evidence of the possible link between the alkylating agent and $\mathrm{Ca}^{2+}$-homeostasis. Thus, our data suggest that Orai1 underexpression might prevent apoptosis triggered either physiologically or by chemotherapies and thus might be important in PCa progression.

Loss of Orai1 function protects LNCaP cells against Tg-induced apoptosis. To further study the role of Orai1 in apoptosis, we used two functional Orai1 mutants described as Isoc inhibitors: the 'selectivity mutant' R91W, which prevents $\mathrm{Ca}^{2+}$ permeation through Orai1, ${ }^{11}$ and the 'coiled-coiled mutant' L273S, which impairs the interaction between STIM1 and Orai1 and thus inhibits its activation. ${ }^{12}$ Electrophysiological measurements on LNCaP cells transiently transfected with the YFP-tagged R91W mutant (YFP-Orai1-R91W) or YFP-tagged L273S mutant (YFPOrai1-L273S) (Figure 4a) revealed a significantly reduced $\mathrm{IP}_{3}$ - and EGTA + BAPTA-evoked $I_{\text {soc }}$ density compared with the control LNCaP cells, consistent with the negative action of these mutants on endogenous Orai1 function.

Moreover, similar to what has been observed with an siRNA-mediated Orai1 knockout, LNCaP cells transfected with any of the Orai1 mutants showed an enhanced resistance to Tg-induced apoptosis. A 24-h exposure to $\mathrm{Tg}$ caused $22 \pm 4 \%$ of apoptosis in the control LNCaP cells, and in the cells transfected with YFP-Orai1-R91W or YFP-Orai1-L273S, 

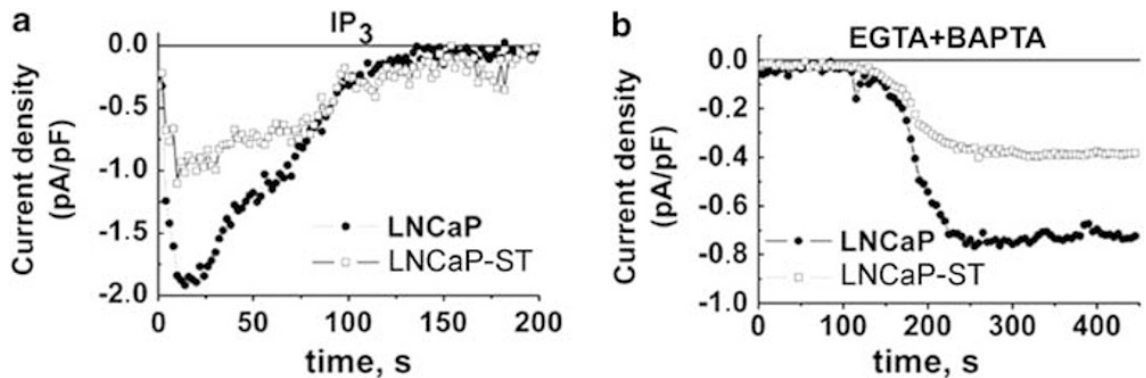

C
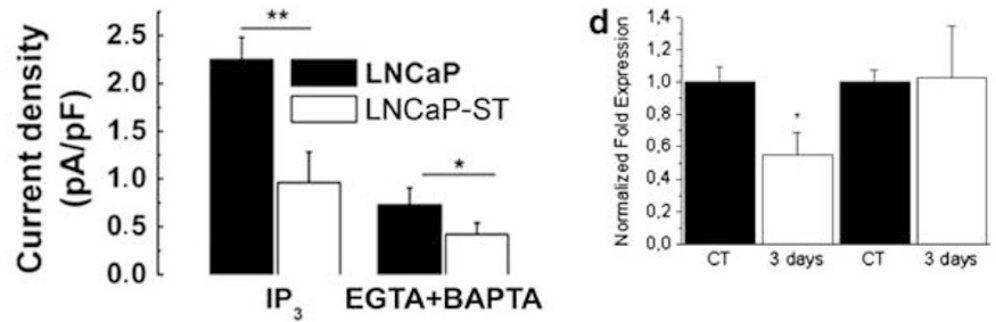

e
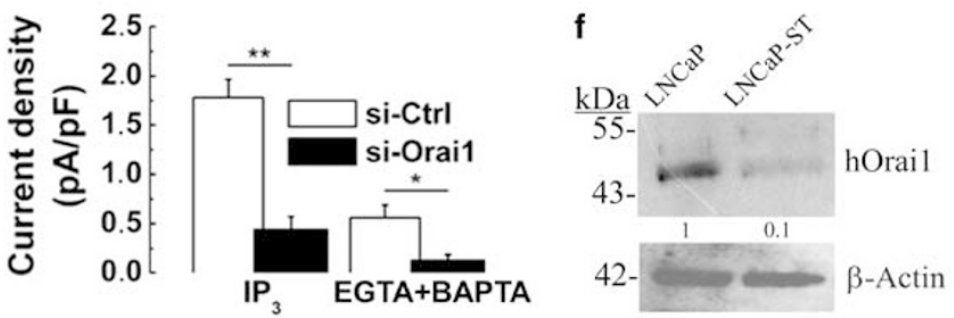

h

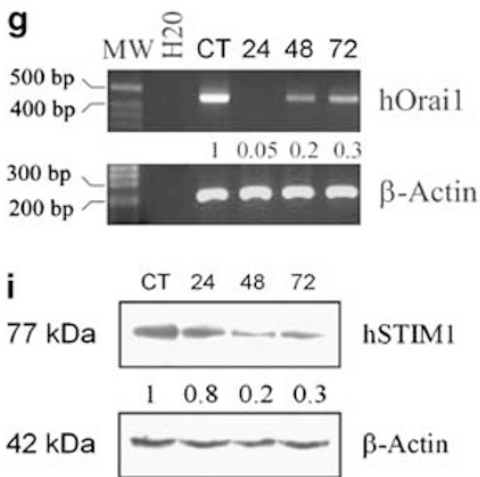

Figure 1 The expression of Orai1 and STIM1 in steroid-deprived prostate cancer epithelial cells and their involvement in SOCE. (a, b) Representative time courses of Isoc development (measured at $-100 \mathrm{mV}$ holding potential) in control LNCaP (black circles) and LNCaP-ST (white squares) cells in response to the dialysis of $100 \mu \mathrm{M}$ IP 3 (a) or $5 \mathrm{mM}$ EGTA (b). (c) Quantification of $\mathrm{IP}_{3^{-}}$and EGTA + BAPTA-induced $I_{\text {soc }}$ densities in control LNCaP (black columns, $2.25 \pm 0.23 \mathrm{pA} / \mathrm{pF}, n=23 \mathrm{and} 0.73 \pm 0.18 \mathrm{pA} / \mathrm{pF}$, $n=31$, respectively) and in LNCaP-ST (white columns, $0.96 \pm 0.32 \mathrm{pA} / \mathrm{pF}, n=17$ and $0.42 \pm 0.12 \mathrm{pA} / \mathrm{pF}, n=21$, respectively) cells. (d) Real-time quantitative PCR showing the expression of Orai1 and STIM1 in control LNCaP cells (CT) and LNCaP cells cultured for 3 days in steroid-deprived medium ( 3 days). (e) Quantification of $\mathrm{IP}_{3^{-}}$and EGTA + BAPTA-induced $I_{\mathrm{soc}}$ densities in LNCaP following $24 \mathrm{~h}$ of transfection with control siRNA (si-Ctrl, white columns, $1.78 \pm 0.18 \mathrm{pA} / \mathrm{pF}, n=23$ and $0.56 \pm 0.13 \mathrm{pA} / \mathrm{pF}$, $n=22$, respectively) and anti-Orai1 siRNA (si-Orai1, black columns, $0.44 \pm 0.13 \mathrm{pA} / \mathrm{pF}, n=19$ and $0.12 \pm 0.06 \mathrm{pA} / \mathrm{pF}, n=22$, respectively). (f) A western blotting experiment showing the relative expression of Orai1 protein upon androgen withdrawal in LNCaP cells. The data are normalized to the expression of $\beta$-actin. (g) Representative RT-PCRs showing the dynamics of Orai1 mRNA expression in LNCaP cells following 24, 48, or $72 \mathrm{~h}$ of transfection with anti-Orai1 siRNA (si-Orai1); CT stands for the cells transfected with control si-RNA; the numbers represent the relative amounts of Orai1 mRNA compared with $\beta$-actin mRNA. (h) Quantification of IP-and EGTA + BAPTA-induced Isoc densities in LNCaP following $24 \mathrm{~h}$ of transfection with control siRNA (si-Ctrl, white columns, $2.52 \pm 0.28 \mathrm{pA} / \mathrm{pF}, n=18$ and $0.70 \pm 0.05 \mathrm{pA} / \mathrm{pF}, n=24$, respectively) and anti-STIM1 siRNA (si-STIM1, black columns, $0.49 \pm 0.23 \mathrm{pA} / \mathrm{pF}, n=18$ and $0.11 \pm 0.03 \mathrm{pA} / \mathrm{pF}, n=23$, respectively). (i) Representative western blot showing the dynamics of the hSTIM1 protein ( $77 \mathrm{kDa}$ ) expression in LNCaP cells following 24,48 or $72 \mathrm{~h}$ of transfection with anti-hSTIM1 siRNA (si-STIM1); CT stands for the cells transfected with control si-RNA; numbers represent relative amounts of hSTIM1 protein compared with $\beta$-actin protein $(42 \mathrm{kDa})$. Throughout the figure, $\left({ }^{*}\right)$ and $\left({ }^{*}\right)$ denote statistically significant differences with $P<0.05$ and $P<0.02$, respectively

the apoptosis rate was decreased by approximately $70-80 \%$ $(6 \pm 3 \%$ and $5 \pm 2 \%$, respectively; Figure $4 b)$.

Taken together, these data indicate that Orai1 forms a major $\mathrm{Ca}^{2+}$ entry pathway required in $\mathrm{Ca}^{2+}$-induced apoptosis: the downregulation or the loss of Orai1 function triggers apoptosis resistance. On the other hand, the Orai1 gain of function by co-expressing both CFP-tagged Orai1 and YFP-tagged STIM1 in LNCaP cells produced a 10-fold 

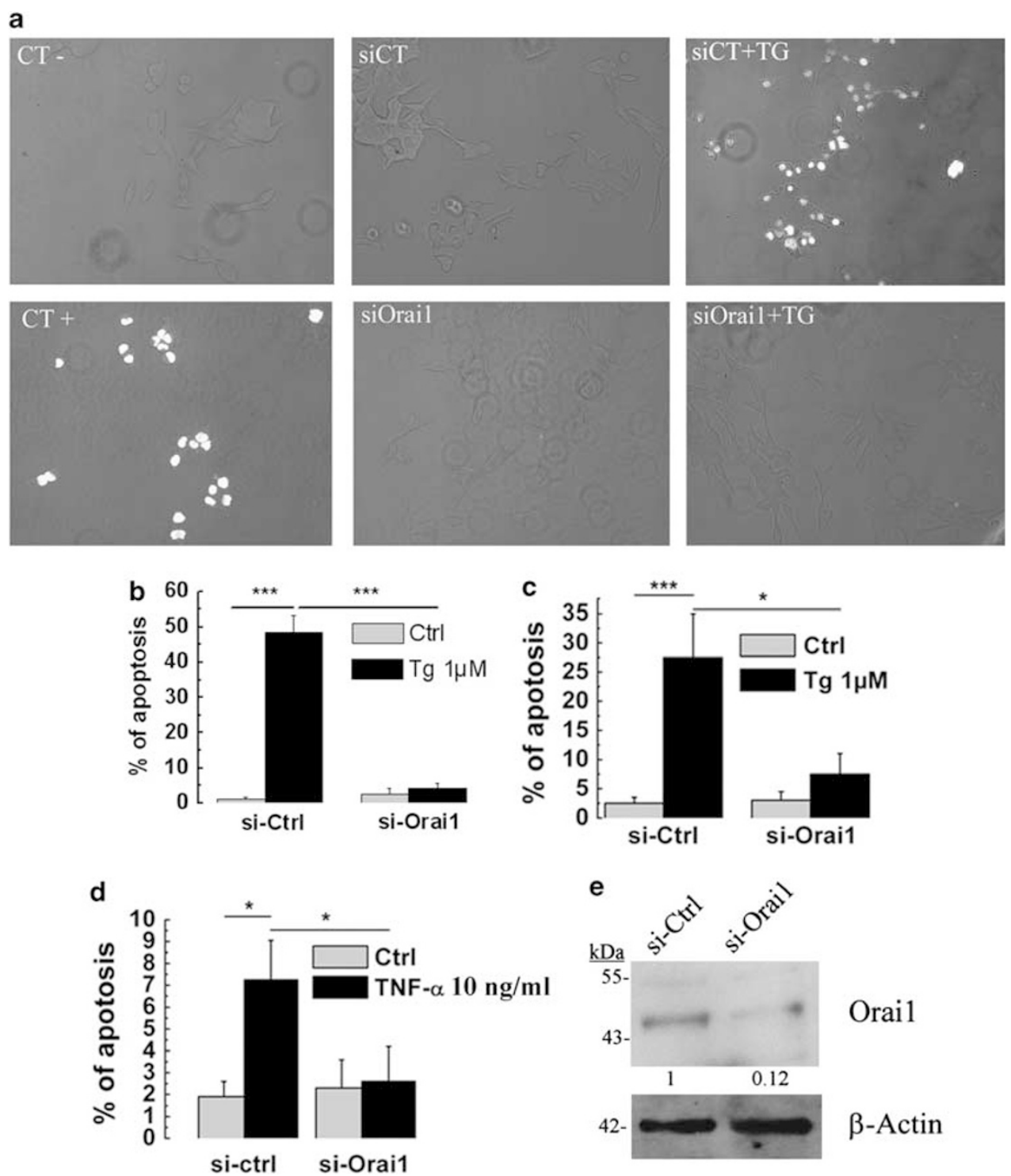

Figure 2 Underexpression of Orai1 protects LNCaP cells against apoptosis. (a) Apoptotic cells revealed using TUNEL technique. (b) Quantification using TUNEL of the apoptosis rate (Ctrl, DMSO-treated, gray columns) and apoptosis induced by $\mathrm{Tg}(1 \mu \mathrm{M}, 24 \mathrm{~h}$, black columns) in control LNCaP cells (si-Ctrl) and LNCaP cells with si-RNAmediated Orai1 silencing (si-Orai1). (c) Quantification using Hoechst technique of the apoptosis rate (Ctrl, DMSO-treated, gray columns) and apoptosis induced by Tg (1 $\mu \mathrm{M}$, $24 \mathrm{~h}$, black columns) in control LNCaP cells (si-Ctrl) and LNCaP cells with si-RNA-mediated Orai1 silencing (si-Orai1). (d) Apoptosis induced by TNF- $\alpha$ (10 ng/ml, $48 \mathrm{~h}$, black columns) in control LNCaP cells (si-Ctrl) and LNCaP cells with si-RNA-mediated Orai1 silencing (si-Orai1) (quantified using Hoechst technique). (e) A representative western blotting of si-Orai1 knockdown in LNCaP cells. The data are normalized to the expression of $\beta$-actin. Throughout the figure, $\left({ }^{*}\right)$ and $\left(^{* *}\right)$ denote statistically significant differences with $P<0.05$ and $P<0.01$, respectively

increase in the $I_{\text {soc }}$ density in response to the infusion of either $\mathrm{IP}_{3}$ or EGTA + BAPTA compared with the respective controls (Figure 4c). At the same time, the co-expression of Orai1 and STIM1 more than doubled the Tg-induced apoptosis $(50 \pm 6 \%$ versus $22 \pm 4 \%$ apoptosis rate; Figure 4d). The control of CFP-tagged Orai1 and YFP-tagged STIM1 transfections into LNCaP cells was performed using confocal microscopy (Figure 4e). Thus, the amplification of SOCE due to Orai1 and STIM1 overexpression correlates with the marked increase in Tg-induced apoptosis.

Orai1 rescue restores $\mathrm{Ca}^{2+}$-induced apoptosis in LNCaP-ST cells: a possible regulation by androgens. We have shown that the decrease in Orai1 expression and the density of $I_{\mathrm{SOC}}$ were responsible for the apoptosis resistance of androgen-deprived LNCaP-ST cells. To further demonstrate the role of Orai1 in apoptosis, we rescued Orai1 expression in LNCaP-ST cells. The cells were transfected with CFP-tagged Orai1. Orai1 overexpression in LNCaP-ST cells resulted in an approximate twofold increase of both $\mathrm{IP}_{3^{-}}$and EGTA + BAPTA-activated $I_{\text {SOC }}$ densities compared with the respective controls (Figure $5 a$ ), indicating that Orai1 overexpression was able, at least in part, to rescue SOCs downregulated by androgen deprivation. This rescue essentially restored the rate of $\mathrm{Tg}$-induced apoptosis 

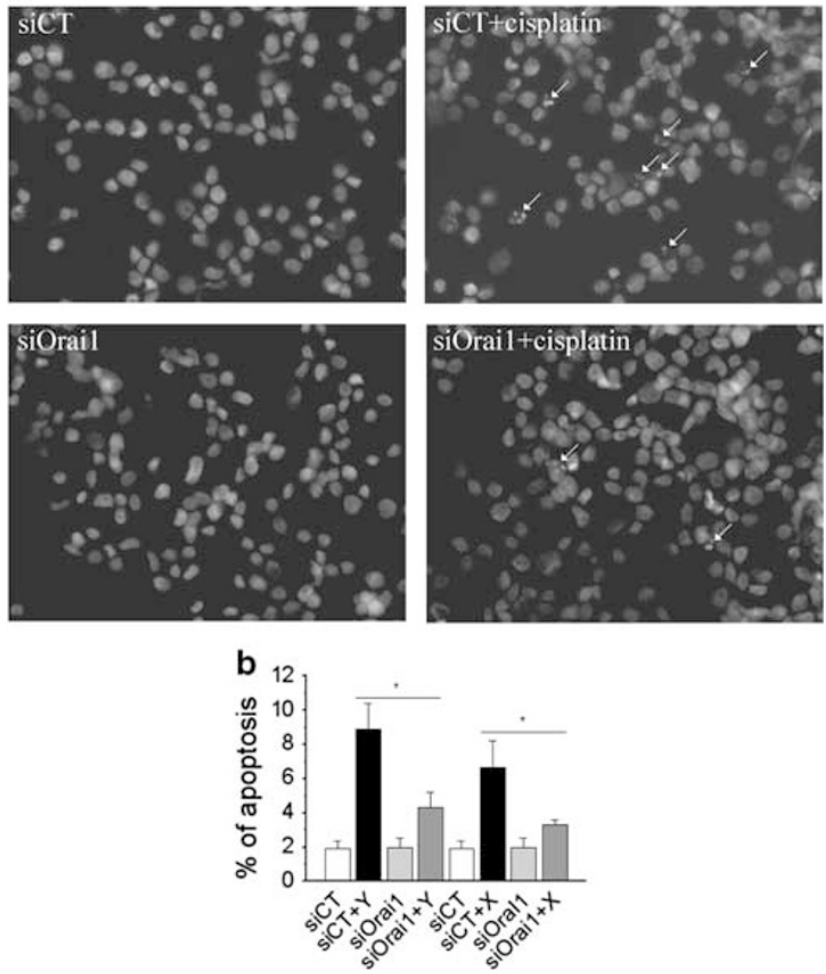

Figure 3 The role of Orai1 in apoptosis induced by alkylating agents cisplatin and oxaliplatin. (a) Representative images of apoptosis rate measured using the Hoechst technique of LNCaP cells treated with $20 \mu \mathrm{M}$ cisplatin. White arrows indicate the apoptotic nuclei. (b) A corresponding histogram showing the apoptosis of $\mathrm{LNCaP}$ cells treated with either $20 \mu \mathrm{M}$ cisplatin $(\mathrm{Y})$ or $40 \mu \mathrm{M}$ oxaliplatin $(\mathrm{X})$ in siCT- and siOrai1-pretreated cells. (*) denotes statistically significant differences with $P<0.05$ (quantified using the Hoechst technique)

of Orai1-overexpressing cells (from $9 \pm 4 \%$ to $16 \pm 6 \%$ ), bringing it closer to the value of normal LNCaP cells (24 $\pm 7 \%$, Figure $5 b)$.

To establish the link between Orai1 and the androgenindependent PCa phenotype, we sought to examine whether the androgen receptor (AR) could regulate Orai1 expression. To directly demonstrate that the orai1 gene is dependent on the functional AR, we used siRNA against AR (si-AR). As shown in Figure $5 \mathrm{~d}$, after $48 \mathrm{~h}$ of siAR transfection, the mRNA level of Orai 1 was decreased by $70 \%$ in the LNCaP cells. Patch-clamp experiments using siAR-transfected cells revealed that their $\mathrm{IP}_{3^{-}}$and EGTA+BAPTA-evoked $I_{\text {Soc }}$ were also reduced by $63 \%$ and $67 \%$, respectively (Figure $5 \mathrm{c}$ ). This result indicates that the $A R$ could regulate Orai1 expression. To further study this potential regulation, we have also used the Matlnspector 7.7.3 program (Genomatix Software $\mathrm{GmbH}$, Munich, Germany) to analyze the putative AR binding sites on Orai1 promoter (see the appropriate section of the discussion).

\section{Discussion}

The appearance of apoptotic resistance in cancer cells is a crucial step for the development and progression of human
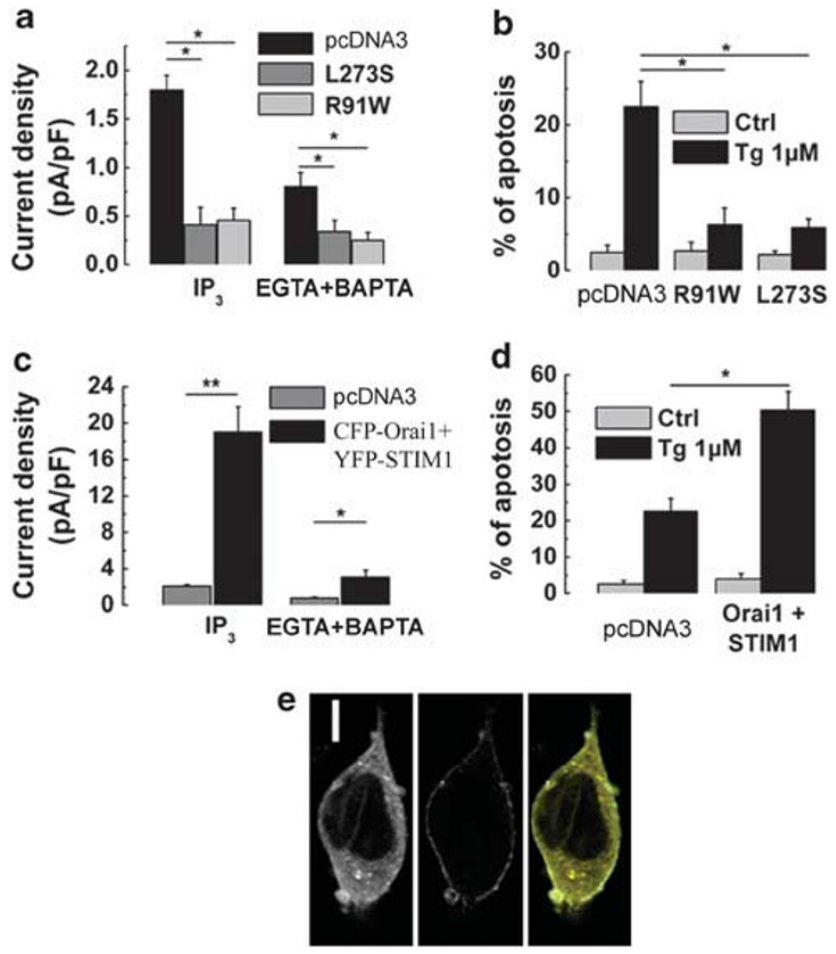

Figure 4 Loss of Orai1 function protects LNCaP cells against thapsigargin (Tg)-induced apoptosis. (a) Quantification of $\mathrm{IP}_{3^{-}}$and EGTA + BAPTA-induced $I_{\mathrm{SOC}}$ densities in control LNCaP cells ( $p c D N A 3$, black columns, $1.8 \pm 0.15 \mathrm{pA} / \mathrm{pF}, n=18$ and $0.8 \pm 0.15 \mathrm{pA} / \mathrm{pF}, n=14$, respectively) and LNCaP cells transfected with YFPOrai1 L273S (dark gray columns, $0.41 \pm 0.18 \mathrm{pA} / \mathrm{pF}, n=18$ and $0.34 \pm 0.12 \mathrm{pA} / \mathrm{pF}$, $n=16$, respectively) or YFP-Orai1 R91W (gray columns, $0.46 \pm 0.12 \mathrm{pA} / \mathrm{pF}, n=18$ and $0.25 \pm 0.08 \mathrm{pA} / \mathrm{pF}, n=16$, respectively). (b) Quantification of the baseline apoptosis (pcDNA3, DMSO-treated, gray columns) and apoptosis induced by $\mathrm{Tg}(1 \mu \mathrm{M}, 24 \mathrm{~h}$, black columns) in control LNCaP cells (Ctrl) and LNCaP cells transfected with Orai1-L273S or Orai1-R91W mutants (quantified using Hoechst technique). (c) Quantification of $\mathrm{IP}_{3}$ - and EGTA + BAPTA-induced Isoc densities in control LNCaP cells (Ctrl, gray columns, $2.1 \pm 0.18 \mathrm{pA} / \mathrm{pF}, n=19$ and $19 \pm 2.8 \mathrm{pA} / \mathrm{pF}, n=32$, respectively) and LNCaP cells co-transfected with YFPSTIM1 and CFP-Orai1 (black columns, $0.75 \pm 0.12 \mathrm{pA} / \mathrm{pF}, \quad n=16$ and $3.1 \pm 0.75 \mathrm{pA} / \mathrm{pF}, n=33$, respectively). (d) Quantification of the baseline apoptosis (pcDNA3, DMSO-treated, gray columns) and apoptosis induced by $\mathrm{Tg}$ $(1 \mu \mathrm{M}, 24 \mathrm{~h}$, black columns) in control LNCaP cells (pcDNA3) and LNCaP cells co-transfected with YFP-STIM1 and CFP-Orai1 (quantified using Hoechst technique). (e) Representative images of LNCaP cell co-transfected with YFPSTIM1 (left) and CFP-Orai1 (middle) and their overlay (right). A white bar in the left panel represents $5 \mu \mathrm{m}$ distance. Throughout the figure, $\left({ }^{*}\right)$ and $\left({ }^{*}\right)$ denote statistically significant differences with $P<0.05$ and $P<0.01$, respectively

PCa to the hormone-refractory androgen-independent phenotype. In the present study, we report three major findings that will allow the understanding of the mechanisms for the acquisition of apoptosis resistance by $\mathrm{PCa}$ cells: (i) the decrease of the endogenous $I_{\mathrm{SOC}}$ is a characteristic feature of the androgen-independent phenotype, caused by the downregulation of the Orai 1 channel; (ii) the downregulation of both Orai 1 expression and $I_{\mathrm{SOC}}$ is used by the PCa cells to develop the apoptosis resistance crucial for PCa development and its progression to the hormone-refractory stage; (iii) Orai1 is a common link between $\mathrm{Ca}^{2+}$ and apoptosis, irrespective of the nature of the apoptosis-triggering stimuli.

The involvement of $\mathrm{Ca}^{2+}$-dependent mechanisms in the induction and regulation of apoptosis is now well established. 

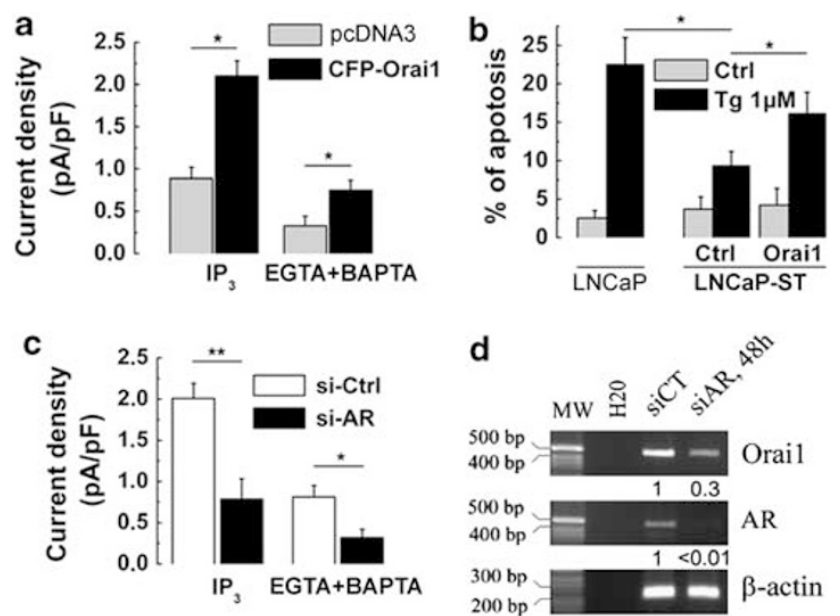

Figure 5 Rescue of Orai1 in androgen-deprived prostate cancer cells abolishes apoptosis resistance. (a) Quantification of $\mathrm{IP}_{3}$ - and EGTA + BAPTA-induced Isoc densities in control LNCaP-ST cells (pcDNA3, gray columns, $0.89 \pm 0.13 \mathrm{pA} / \mathrm{pF}$, $n=13$ and $0.33 \pm 0.11 \mathrm{pA} / \mathrm{pF}, n=14$, respectively) and LNCaP-ST cells transfected with CFP-Orai1 (black columns, $2.1 \pm 0.18 \mathrm{pA} / \mathrm{pF}, n=21$ and $0.75 \pm 0.12 \mathrm{pA} / \mathrm{pF}, n=23$, respectively). (b) Quantification of the baseline apoptosis (pcDNA3, DMSO-treated, gray columns) and apoptosis induced by $\mathrm{Tg}$ ( $1 \mu \mathrm{M}, 24 \mathrm{~h}$, black columns) in control LNCaP cells and in LNCaP-ST cells with or without Orai1 rescued by transfection with CFP-Orai1 (quantified using the Hoechst technique). (c) Quantification of $I P_{3}$ - and EGTA + BAPTA-induced $I_{\text {soc }}$ densities in LNCaP following $48 \mathrm{~h}$ of transfection with control siRNA (si-Ctrl, white columns, $2.01 \pm 0.19 \mathrm{pA} / \mathrm{pF}, n=19$ and $0.78 \pm 0.25 \mathrm{pA} / \mathrm{pF}, n=18$, respectively) and antiAR siRNA (si-AR, black columns, $0.81 \pm 0.14 \mathrm{pA} / \mathrm{pF}, n=21$ and $0.32 \pm 0.10 \mathrm{pA} / \mathrm{pF}$, $n=15$, respectively). (d) Representative RT-PCRs showing changes in Orai1 and androgen receptor (AR) mRNA expression in LNCaP cells following $48 \mathrm{~h}$ of transfection with anti-AR siRNA (si-AR) compared with the cells transfected with control si-RNA (CT); numbers represent relative amounts of Orai1 and AR mRNA compared with $\beta$-actin mRNA. Throughout the figure, $\left({ }^{*}\right)$ and $\left({ }^{* *}\right)$ denote statistically significant differences with $P<0.05$ and $P<0.02$, respectively

Alterations in the ER storage capacity and SOC activity seem to have a major role in the establishment of an androgenindependent apoptosis-resistant phenotype of $\mathrm{PCa}$ cells. Indeed, as we have shown in our previous works on androgen-independent, apoptosis-resistant phenotypes of LNCaP PCa cells (such as androgen-deprived LNCaP cells, $\mathrm{LNCaP}$ cells overexpressing the anti-apoptotic Bcl-2 protein and neuroendocrine-differentiated LNCaP cells), increased resistance to Tg- and TNF- $\alpha$-induced apoptosis is characterized by (i) the reduced basal $\mathrm{Ca}^{2+}$ filling of the ER pool and (ii) reduced store-operated $\mathrm{Ca}^{2+}$ entry. ${ }^{5,13}$ The primary trigger for apoptosis in androgen-dependent cells is ER store depletion and a sustained $\mathrm{Ca}^{2+}$ influx may not even be required. ${ }^{8}$ Interestingly, for androgen-independent cells, ER store depletion per se is not sufficient to induce cell death without the lethal $\mathrm{Ca}^{2+}$ influx from SOCE. ${ }^{2,9,14}$ Therefore, the identification of the molecular nature of SOC and their activation/regulation mechanisms are of great importance for controlling androgen-independent PCa cell apoptosis.

During recent years, a new molecular candidate for SOC termed Orai1 has been identified and characterized. Orai1 mediates CRAC currents and SOCE in a large variety of cells and is involved in a wide range of cell functions, including endothelial cell proliferation, ${ }^{15}$ lymphocyte proliferation, ${ }^{16}$ mast cell activation, ${ }^{13}$ as well as skeletal muscle development and a contractile function. ${ }^{17}$ However, despite the suggested pivotal role of SOCs in the apoptosis resistance of PCa cells, the involvement of Orai1 in prostate-specific SOC, as well as in $\mathrm{Ca}^{2+}$-dependent apoptosis of PCa cells, has never been studied.

In the present study, we have shown that Orai1, an ion channel in the PM, and STIM1, as a signal transducer from the $E R$, represent the major molecular components of SOCE in PCa epithelial cells: the siRNA-mediated knockout of any of them strongly diminishes $I_{\mathrm{SOC}}$ in LNCaP cells. However, as only Orai1 expression was found to decrease in LNCaP cells following androgen deprivation, we hypothesized that the downregulation of $I_{S O C}$, which follows the transition of $\mathrm{PCa}$ cells to androgen independence and apoptosis resistance, is associated, at least in part, with the reduction of Orai1 levels.

Androgen ablation therapy in prostate adenocarcinoma induces an involution of prostate tissue mainly through the enhancement of cellular apoptosis. ${ }^{18}$ However, a subset of malignant cells emerges as a new population of apoptosisresistant cells. The enrichment of the prostate with such cell phenotypes eventually causes virtually all tumors to relapse into an androgen-independent, more aggressively growing type. $^{18}$

Androgens have an essential role in prostate carcinogenesis and androgen independency of the most malignant androgen-independent phenotype, which is known to arise from loss of the AR (e.g., Bonkhoff ${ }^{19}$ ). Therefore, we assumed that the decrease in Orai 1 expression and $I_{S O C}$ density following the induction of LNCaP cell differentiation by androgen withdrawal occurs because orai1 gene expression might be regulated by the functional AR. Our data showed that AR silencing in LNCaP cells leads to a dramatic decrease in Orai1 expression as well as in $I_{\text {soc }}$ density. The structure of the promoter of such a classic androgen-dependent gene as the prostate-specific antigen usually includes an androgenresponsive element (ARE) close to the transcriptional start site and other AREs located several kilobase pairs upstream within the enhancers. ${ }^{20}$ In order to estimate a possible orai1 gene regulation by $A R$, we studied the human Orai1 promoter sequence. The genomic sequence corresponding to $6200 \mathrm{bp}$ upstream and $100 \mathrm{bp}$ downstream of hOrai1 ATG was used for the transcription factor analysis. The Matlnspector 7.7.3 program was used to analyze the putative AR binding sites. ${ }^{21}$ The orai1 promoter sequence was analyzed for the presence of AREs using a prostate-specific matrix, which is associated with transcription factors that are expressed and transcriptionally active in this tissue. We identified several AREs: three of them located at $-3700,-4105$ and $-4700 \mathrm{bp}$ from the Orai1 ATG codon with more than $80 \%$ of matrix similarity. These results may suggest that Orai1 is probably an androgen-responsive gene in the prostate. Moreover, given that Orai1 represents a key component of prostate-specific SOC, we hypothesized that the decrease of functional SOCs during PCa progression to the aggressive androgen-independent stage results from downregulation of the functional AR and ultimately the deregulation of Orai1.

The transition to the androgen-independent phenotype not only influences Orai1 expression and the SOCE, but, more importantly, the acquisition of apoptosis resistance also. 
We have found that susceptibility of the cells to the induction of $\mathrm{Ca}^{2+}$-dependent apoptosis was always in direct correlation with Orai1 expression and $I_{\text {SOC }}$ density: the lower the current density (i.e., low Orai1 expression), the higher the apoptosis resistance is (i.e., low apoptosis rate). This correlation existed independently of the experimental tools used to reduce Orai1 expression and activity: the androgen-dependence status of the LNCaP cells (i.e., androgen deprivation or AR silencing), the targeted downregulation of Orai1 (anti-Orai1 siRNA) or Orai1 mutants. Interestingly, the pro-apoptotic effects of Orai1 were independent of the apoptosis-inducing stimuli. Orai 1 downregulation is likely to diminish the sustained cytosolic $\mathrm{Ca}^{2+}$ increase and protect PCa cells from apoptosis. We first used a classical $\mathrm{Ca}^{2+}$-dependent apoptosis inducer, $\mathrm{Tg}$. Tg is a very powerful tool and as such was even recently proposed to be used as a 'smart bomb' to target androgen-independent $\mathrm{PCa}^{22}$ Our data demonstrate that Tg-evoked apoptosis was predominantly mediated via Orai 1 activation and the efficient knockdown of the latter may be an important means to protect the cell from eventual death. To distinguish between direct and indirect $\mathrm{Ca}^{2+}$-dependent apoptosis, we used another apoptosis inducer, $\mathrm{TNF} \alpha$, a pro-inflammatory cytokine that deregulates $\mathrm{Ca}^{2+}$ homeostasis by inducing $\mathrm{ER}$ stress. According to our previous observations, TNF $\alpha$ is an effective pro-apoptotic agent of PCa epithelial cells. ${ }^{9}$ Indeed, TNF $\alpha$ induced apoptosis of the LNCaP cells; however, even this non-direct $\mathrm{Ca}^{2+}$-dependent mechanism was significantly blocked by the Orai1 knockdown, suggesting that Orai1mediated $\mathrm{Ca}^{2+}$ entry has a major role in apoptosis, especially in its early phase. Furthermore, we used two other strong apoptosis inducers: cisplatin and oxaliplatin. These alkylating agents react in vivo by binding to and causing crosslinking of DNA, which ultimately triggers apoptosis (for a review, see Gonzalez et $a l^{23}$ ). Cisplatin-induced DNA damage seems to be a long and complex process of cell death and because cisplatin is a nonspecific drug that reacts not only with DNA but also with proteins, there is a possibility that an easier process of initiation, such as damage to cytoplasmic proteins, may take place. ${ }^{23}$ Moreover, inhibition of the proteasome induces ER stress and also activates the unfolded protein response, thereby triggering apoptosis. ${ }^{24}$ Cisplatin has also been shown to stimulate ER stress and increase ER dilation, intracellular $\mathrm{Ca}^{2+}$ levels, and cell death. ${ }^{25}$ This eventually links cisplatin with ER stress and increased intracellular $\mathrm{Ca}^{2+}$ levels, which may be successfully inhibited by Orai1 knockdown with the consecutive downregulation of $I_{\mathrm{SOC}}$ and apoptosis in general. In fact, we have previously shown that ER stress induces $\mathrm{Ca}^{2+}$ entry, ${ }^{26}$ and we observed that $24-\mathrm{h}$ pretreatment with either cisplatin or oxaliplatin increases the cytoplasmic $\left[\mathrm{Ca}^{2+}\right]$.

Finally, recent studies, concerning Orai1 activation, have shown that the overexpression of both Orai1 and STIM1 induces a 20 -fold increase in the $I_{\text {soc }}$, resulting in a 'monster CRAC'. ${ }^{27}$ The gain of SOC function in the androgendependent LNCaP cells in response to the overexpression of Orai1 and STIM1 was manifested by the appearance of a high-amplitude $I_{\text {soc }}$, which led to the marked enhancement of $\mathrm{Ca}^{2+}$-dependent apoptosis. Moreover, the rescue of the $I_{\mathrm{SOC}}$ function by Orai1 overexpression in androgen-deprived apoptosis-resistant LNCaP cells is able to restore the

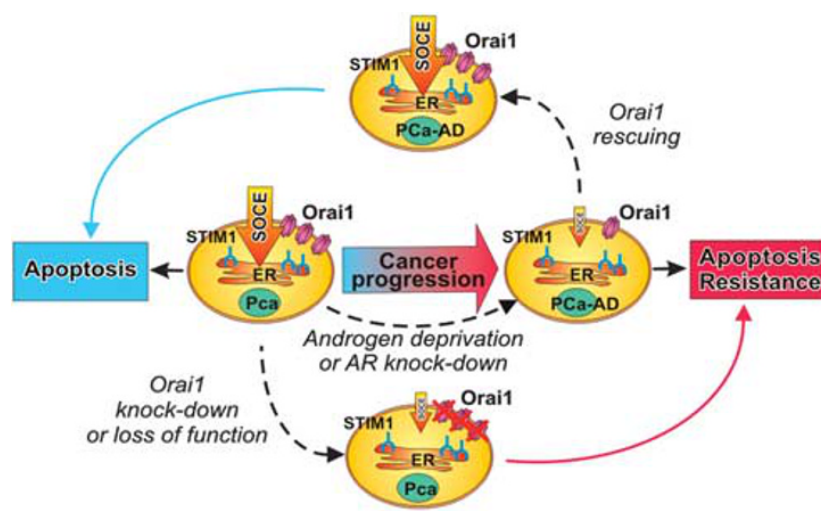

Figure 6 Schematic diagram summarizing the principal findings of this study. The progression to androgen-independent $\mathrm{PCa}$ is associated with the appearance of new cell phenotypes characterized by decreased SOCE due to the downregulation of Orai1, which results in enhanced resistance to $\mathrm{Ca}^{2+}$-dependent apoptosis. This natural process, which occurred in the in vivo tumors, can be mimicked by the artificial silencing of Orai1 expression or inhibition of Orai1 function with dominant-negative mutants. On the other hand, rescuing Orai1 in androgenindependent PCa cell phenotypes can restore the normal rate of $\mathrm{Ca}^{2+}$-dependent apoptosis, thus providing the means for the perspective therapies of advanced $\mathrm{PCa}$

apoptosis rate close to that of androgen-dependent $\mathrm{PCa}$ cells. This result is especially impressive, as it is relevant to the development of perspective therapies for advanced, androgen-independent PCas.

Thus, we have shown the involvement of Orai1 as a principal molecular component of native SOCs in the $\mathrm{Ca}^{2+}$-dependent apoptosis of $\mathrm{PCa}$ cells. Our results conclude that the transition to the androgen-independent $\mathrm{PCa}$ phenotype is associated with the loss of Orai1 expression leading to the downregulation of SOCE, which precludes cytosolic $\mathrm{Ca}^{2+}$ increases that are sufficient enough to induce apoptosis via well-known mitochondrial and cytosolic mechanisms. In addition, reduced SOCE may contribute to the chronic underfilling of $\mathrm{ER} \mathrm{Ca}^{2+}$ stores, which represent the new state of equilibrium for androgen-independent $\mathrm{PCa}$ cells, ${ }^{2}$ further enhancing their apoptosis resistance (Figure 6).

Our data are consistent with the notion that SOCE and Orai1 are important players in apoptosis induction. However, it seems that apoptosis is not the only process directly related to SOCE. A recent study revealed that Orai1/STIM1 and SOCE are also essential for breast tumor cell migration, invasion and metastasis. ${ }^{28}$ It also has been shown that the SOCE and STIM1/Orai1 are involved in migration, proliferation, ${ }^{29}$ and cell cycle progression. ${ }^{30}$ Thus, Orai1/STIM1 and SOCE seem to have important roles in the wide spectrum of $\mathrm{Ca}^{2+}$-dependent cancer-related behaviors, and are expected to have a significant impact on future research.

\section{Materials and Methods}

Cell culture. The androgen-dependent human PCa cell line LNCaP was obtained from the American Type Culture Collection (ATCC, Manassas, VA, USA) and maintained in culture in RPMI 1640 medium (Gibco-Life Technologies, Carlsbad, CA, USA) supplemented with 10\% fetal calf serum (Seromed, Poly-Labo, Strasbourg, France), $5 \mathrm{mM}$ L-glutamine (Sigma, L'Isle d'Abeau, France), and kanamycin $100 \mu \mathrm{g} / \mathrm{ml}$. Charcoal-striped fetal calf serum (10\%) was added to phenol red-free RPMI medium together with kanamycin and L-glutamine (as above) to 
Table 1 List of primers used for Q-PCR assays (1-5) and siRNA sequences targeting hOrai1(6) or hSTIM1 (7)

\begin{tabular}{|c|c|c|c|c|}
\hline No. & Name (accession no.) & Forward $\left(5^{\prime}-\ldots-3^{\prime}\right)$ & Backward $\left(5^{\prime}-\ldots-3^{\prime}\right)$ & Expected product size (bp) \\
\hline $\begin{array}{l}1 . \\
2 . \\
3 . \\
4 . \\
5 . \\
6 . \\
7 .\end{array}$ & $\begin{array}{l}\text { hSTIM1 (NM_003156) } \\
\text { hOrai1 (NM_032790) } \\
\text { HPRT (NM_000194) } \\
\text { hOrai1 (NM_032790) } \\
\beta \text {-Actin (NM_001101) } \\
\text { hOrai1 siRNA } \\
\text { hSTIM1 siRNA }\end{array}$ & $\begin{array}{l}\text { TGTGGAGCTGCCTCAGTATG } \\
\text { ATGGTGGCAATGGTGGAG } \\
\text { GGCGTCGTGATTAGTGATGAT } \\
\text { CTTCAGTGCCTGCACCACAG } \\
\text { CAGAGCAAGAGAGGCATCCT } \\
\text { 5'-UGAGCAACGU } \\
\text { 5'-GGCUCUGGAU }\end{array}$ & $\begin{array}{l}\text { CTTCAGCACAGTCCCTGTCA } \\
\text { CTGATCATGAGCGCAAACAG } \\
\text { CGAGCAAGACGTTCAGTCCT } \\
\text { CCTGGAACTGTCGGTCAGTC } \\
\text { GTTGAAGGTCTCAAACATGATC } \\
\text { ACAAUCU (dTdT)-3' } \\
\text { AGUGCUC (dTdT)--3' }\end{array}$ & $\begin{array}{l}112 \\
115 \\
134 \\
450 \\
209\end{array}$ \\
\hline
\end{tabular}

Sequences for the Q-PCR are indicated in italic script.

create steroid-deprived conditions. Cells were grown at $37^{\circ} \mathrm{C}$ in a humidified atmosphere containing $5 \% \mathrm{CO}_{2}$. The medium was replaced every $48 \mathrm{~h}$. For the experiments, cells were seeded in six-well plates for PCR and western blotting, 35-mm culture for the patch-clamp experiments, and onto glass coverslips for the confocal microscopy.

Electrophysiology and solutions. Macroscopic currents were recorded from LNCaP cells in the whole-cell configuration of the patch-clamp technique using a computer-controlled EPC-9 amplifier (HEKA Electronic, Lambrecht/Pfalz, Germany), as previously described. ${ }^{26}$

The composition of the extracellular solution for patch-clamp recording was (in mM): $120 \mathrm{NaCl}, 5 \mathrm{KCl}, 10 \mathrm{CaCl}_{2}, 2 \mathrm{MgCl}_{2}, 5$ glucose, and $10 \mathrm{HEPES}, \mathrm{pH} 7.4$ (adjusted with TEA-OH), osmolarity $310 \mathrm{mOsm} / \mathrm{kg}$ adjusted with D-mannitol. The patch pipettes were filled with the basic intracellular pipette solution (in $\mathrm{mM}$ ): 120 Cs-methane sulfonate, $10 \mathrm{CsCl}, 10 \mathrm{HEPES}, 10 \mathrm{BAPTA}$ (1.2-bis(2-aminophenoxy)ethane $N, N, N^{\prime}, N^{\prime}$-tetraacetic acid), and $6 \mathrm{MgCl}_{2}(\mathrm{pH}$ adjusted to 7.4 with $\mathrm{CsOH}$ and osmolarity $295 \mathrm{mOsm} / \mathrm{kg}$ adjusted with D-mannitol). The necessary supplements in the desired concentrations were added to the experimental solutions directly from the appropriate stock solutions, dissolved in water, ethanol or dimethylsulfoxide. All chemicals were purchased from Sigma-Aldrich (St. Louis, MO, USA). In the course of the patch-clamp recording, drugs and solutions were applied to the cells through the multiline microperfusion system with common outflow (Cell MicroControls, Norfolk, VA, USA) placed in close proximity $(\sim 200 \mu \mathrm{m})$ to the studied cell. The experiments were carried out at room temperature.

Western blotting. LNCaP cells were treated with an ice-cold lysis buffer containing $10 \mathrm{mM}$ Tris- $\mathrm{HCl}, \mathrm{pH} 7.4,150 \mathrm{mM} \mathrm{NaCl}, 10 \mathrm{mM} \mathrm{MgCl}, 1 \mathrm{mM}$ PMSF, $1 \%$ Nonidet P-40, and protease inhibitor cocktail from Sigma. The lysates were centrifuged at $15000 \times g$ and $4^{\circ} \mathrm{C}$ for $20 \mathrm{~min}$, mixed with a sample buffer containing $125 \mathrm{mM}$ Tris- $\mathrm{HCl}, \mathrm{pH} 6.8,4 \%$ SDS, $5 \% \beta$-mercaptoethanol, $20 \%$ glycerol, and $0.01 \%$ bromophenol blue, and boiled for $5 \mathrm{~min}$ at $95^{\circ} \mathrm{C}$. The total protein samples were subjected to $8-10 \%$ SDS-PAGE and transferred to a nitrocellulose membrane by semi-dry western blotting (Bio-Rad Laboratories, Hercules, CA, USA). The membrane was blocked in a 5\% milk TNT buffer (Tris- $\mathrm{HCl}, \mathrm{pH} 7.4,140 \mathrm{mM} \mathrm{NaCl}$, and $0.05 \%$ Tween 20 ) overnight, and then probed using a specific rabbit polyclonal anti-Orai1 antibody (1:200, ProScience, Poway, CA, USA) anti-mouse monoclonal ant-STIM1 antibody (1:250, BD Biosciences, Mountain View, CA, USA) and anti- $\beta$ actin (Lab Vision Co., Fremont, CA, USA, 1/1000) antibodies. The bands on the membrane were visualized by enhanced chemiluminescence (Pierce Biotechnologies Inc., Fremont, CA, USA). A densitometric analysis was performed using a Bio-Rad image acquisition system (Bio-Rad Laboratories).

RT-PCR. RT-PCR experiments were performed as previously described. ${ }^{31}$ DNA amplification conditions included the initial denaturation step of $7 \mathrm{~min}$ at $95^{\circ} \mathrm{C}$, and 36 cycles of $30 \mathrm{~s}$ at $95^{\circ} \mathrm{C}, 30 \mathrm{~s}$ at $60^{\circ} \mathrm{C}$, and $30 \mathrm{~s}$ at $72^{\circ} \mathrm{C}$, and finally 7 min at $72^{\circ} \mathrm{C}$. Primers used are listed in Table 1.

siRNA transfection. LNCaP cells were transfected with $50 \mathrm{nM}$ of siRNA against Orai1, STIM1, and AR (Dharmacon Inc., Fremont, CA, USA) using $6 \mu \mathrm{l}$ Hyperfect transfection reagent (Qiagen Inc., Courtaboeuf, France), following the manufacturer's instructions (see Table 1 for the siRNA sequences).

Nucleofection. The transfection of $\mathrm{LNCaP}$ with different plasmids was carried out using Nucleofector (Amaxa GmbH, Köln, Germany) according to the manufacturer's instructions. In brief, $2 \mu \mathrm{g}$ of the plasmid was transfected into 2 million trypsinized cells, which were then plated onto six-well dishes, $35-\mathrm{mm}$ dishes or onto glass coverslips for $48 \mathrm{~h}$.

Immunocytochemistry. Cells grown on glass coverslips were washed once with PBS and fixed in $3.5 \%$ paraformaldehyde in PBS. Fluorescence analysis was carried out using a Carl Zeiss LSM 510 connected to a Zeiss Axiovert 200M with $63 \times 1.4$ numerical aperture oil immersion lens at room temperature. Both channels were excited, collected separately, and then merged to examine colocalization using Carl Zeiss LSM Image Examiner software (Le Pecq, France).

Apoptosis assay. The level of apoptosis was estimated from the number of apoptotic nuclei revealed either by TUNEL assay (Roche Biochemicals, Burlington, NC, USA) or by Hoechst staining. The percentage of apoptotic cells was determined by counting at least five random fields for each condition done in triplicate for each $n$ (the detailed procedure has been described previously). ${ }^{8}$

Quantitative real-time PCR. The quantitative real-time PCR of Orai1, STIM1, and HPRT mRNA transcript was done using MESA GREEN qPCR MasterMix Plus for SYBR Assay (Eurogentec, Angers, France) on the Biorad CFX96 Real-Time PCR Detection System. The sequences of primers are indicated in Table 1. The HPRT gene was used as an endogenous control to normalize variations in the RNA extractions, the degree of RNA degradation, and variability in RT efficiency. To quantify the results, we used the comparative threshold cycle method described by Livak and Schmittgen. ${ }^{32}$

Cloning of Orai1 and STIM1 and the site-directed mutagenesis. All of the procedures used to clone Orai1 and STIM1 and to create the two Orai1 mutants, the 'selectivity mutant' R91W and the 'coiled-coil mutant' L273S, have been previously described in detail. ${ }^{12}$

Data analysis. For each type of experiment, data were accumulated from at least five measurements. Electrophysiological data were analyzed offline using HEKA (HEKA Electronic) and Origin 7.0 (Microcal Software Inc., Northampton, MA, USA) software. The results were expressed as mean \pm S.E.M., where appropriate. A Student's $t$-test was used for the statistical comparison of the differences, with $P<0.05$ considered as significant. In the graphs, $\left({ }^{*}\right)$ and $\left({ }^{* *}\right)$ denote statistically significant differences of $P<0.05$ and $P<0.01$, respectively.

\section{Conflict of interest}

The authors declare no conflict of interest.

Acknowledgements. This work was supported by grants from the Institut National de la Santé et de la Recherche Médicale (INSERM), Ministère de l'Education Nationale et Ligue Nationale Contre le Cancer.

1. Scorrano L, Oakes SA, Opferman JT, Cheng EH, Sorcinelli MD, Pozzan T et al. BAX and BAK regulation of endoplasmic reticulum $\mathrm{Ca2t:}$ a control point for apoptosis. Science 2003; 300: 135-139.

2. Prevarskaya N, Skryma R, Shuba Y. Ca2+ homeostasis in apoptotic resistance of prostate cancer cells. Biochem Biophys Res Commun 2004; 322: 1326-1335. 
3. Pinton P, Giorgi C, Siviero R, Zecchini E, Rizzuto R. Calcium and apoptosis: ER mitochondria Ca2+ transfer in the control of apoptosis. Oncogene 2008; 27: 6407-6418.

4. Norberg E, Gogvadze V, Ott M, Horn M, Uhlen P, Orrenius S et al. An increase in intracellular $\mathrm{Ca} 2+$ is required for the activation of mitochondrial calpain to release AIF during cell death. Cell Death Differ 2008; 15: 1857-1864.

5. Fixemer T, Remberger K, Bonkhoff H. Apoptosis resistance of neuroendocrine phenotypes in prostatic adenocarcinoma. Prostate 2002; 53: 118-123.

6. Raffo AJ, Perlman H, Chen MW, Day ML, Streitman JS, Buttyan R. Overexpression of bcl-2 protects prostate cancer cells from apoptosis in vitro and confers resistance to androgen depletion in vivo. Cancer Res 1995; 55: 4438-4445.

7. Tannock IF, de Wit R, Berry WR, Horti J, Pluzanska A, Chi KN et al. Docetaxel plus prednisone or mitoxantrone plus prednisone for advanced prostate cancer. N Engl J Med 2004; 351: 1502-1512.

8. Skryma R, Mariot $\mathrm{P}$, Bourhis $\mathrm{XL}$, Coppenolle FV, Shuba $\mathrm{Y}$, Vanden Abeele $\mathrm{F}$ et al Store depletion and store-operated $\mathrm{Ca} 2+$ current in human prostate cancer $\mathrm{LNCaP}$ cells: involvement in apoptosis. J Physiol 2000; 527 (Pt 1): 71-83.

9. Vanoverberghe K, Vanden Abeele F, Mariot P, Lepage G, Roudbaraki M, Bonnal JL et al. $\mathrm{Ca} 2+$ homeostasis and apoptotic resistance of neuroendocrine-differentiated prostate cancer cells. Cell Death Differ 2004; 11: 321-330.

10. Zhang SL, Yu Y, Roos J, Kozak JA, Deerinck TJ, Ellisman MH et al. STIM1 is a Ca2+ sensor that activates CRAC channels and migrates from the Ca2+ store to the plasma membrane. Nature 2005; 437: 902-905.

11. Liao Y, Erxleben C, Yildirim E, Abramowitz J, Armstrong DL, Birnbaumer L. Orai proteins interact with TRPC channels and confer responsiveness to store depletion. Proc Natl Acad Sci USA 2007; 104: 4682-4687.

12. Muik M, Frischauf I, Derler I, Fahrner M, Bergsmann J, Eder P et al. Dynamic coupling of the putative coiled-coil domain of ORAI1 with STIM1 mediates ORAl1 channel activation. J Biol Chem 2008; 283: 8014-8022.

13. $\mathrm{Ng} \mathrm{SW}$, di Capite J, Singaravelu K, Parekh AB. Sustained activation of the tyrosine kinase Syk by antigen in mast cells requires local $\mathrm{Ca} 2+$ influx through $\mathrm{Ca} 2+$ release-activated Ca2+ channels. J Biol Chem 2008; 283: 31348-31355.

14. Vanden Abeele F, Skryma R, Shuba Y, Van Coppenolle F, Slomianny C, Roudbaraki M et al. Bcl-2-dependent modulation of $\mathrm{Ca}(2+)$ homeostasis and store-operated channels in prostate cancer cells. Cancer Cell 2002; 1: 169-179.

15. Abdullaev F, Bludov YV, Dmitriev SV, Kevrekidis PG, Konotop VV. Generalized neighborinteraction models induced by nonlinear lattices. Phys Rev E Stat Nonlin Soft Matter Phys 2008; 77 (Pt 2): 016604

16. Gwack Y, Feske S, Srikanth S, Hogan PG, Rao A. Signalling to transcription: store-operated $\mathrm{Ca}(2+)$ entry and NFAT activation in lymphocytes. Cell Calcium 2007; 42: 145-156.

17. Stiber J, Hawkins A, Zhang ZS, Wang S, Burch J, Graham V et al. STIM1 signalling controls store-operated calcium entry required for development and contractile function in skeletal muscle. Nat Cell Biol 2008; 10: 688-697.

18. Denmeade SR, Lin XS, Isaacs JT. Role of programmed (apoptotic) cell death during the progression and therapy for prostate cancer. Prostate 1996; 28: 251-265.

19. Bonkhoff $\mathrm{H}$. Neuroendocrine differentiation in human prostate cancer. Morphogenesis, proliferation and androgen receptor status. Ann Oncol 2001; 12: S141-S144.
20. Nantermet PV, Xu J, Yu Y, Hodor P, Holder D, Adamski S et al. Identification of genetic pathways activated by the androgen receptor during the induction of proliferation in the ventral prostate gland. J Biol Chem 2004; 279: 1310-1322.

21. Cartharius K, Frech K, Grote K, Klocke B, Haltmeier M, Klingenhoff A et al. MatInspector and beyond: promoter analysis based on transcription factor binding sites. Bioinformatics 2005; 21: 2933-2942.

22. Denmeade SR, Isaacs JT. The SERCA pump as a therapeutic target: making a 'smart bomb' for prostate cancer. Cancer Biol Ther 2005; 4: 14-22.

23. Gonzalez VM, Fuertes MA, Alonso C, Perez JM. Is cisplatin-induced cell death always produced by apoptosis? Mol Pharmacol 2001; 59: 657-663.

24. Di Sano F, Ferraro E, Tufi R, Achsel T, Piacentini M, Cecconi F. Endoplasmic reticulum stress induces apoptosis by an apoptosome-dependent but caspase 12-independent mechanism. J Biol Chem 2006; 281: 2693-2700.

25. Nawrocki ST, Carew JS, Dunner Jr K, Boise LH, Chiao PJ, Huang P et al. Bortezomib inhibits PKR-like endoplasmic reticulum (ER) kinase and induces apoptosis via ER stress in human pancreatic cancer cells. Cancer Res 2005; 65: 11510-11519.

26. Flourakis M, Van Coppenolle F, Lehen'kyi V, Beck B, Skryma R, Prevarskaya N. Passive calcium leak via translocon is a first step for iPLA2-pathway regulated store operated channels activation. FASEB J 2006; 20: 1215-1217.

27. Peinelt $C$, Vig M, Koomoa DL, Beck A, Nadler MJ, Koblan-Huberson $M$ et al. Amplification of CRAC current by STIM1 and CRACM1 (Orai1). Nat Cell Biol 2006; 8: 771-773.

28. Yang S, Zhang JJ, Huang XY. Orai1 and STIM1 are critical for breast tumor cell migration and metastasis. Cancer Cell 2009; 15: 124-134.

29. Potier M, Gonzalez JC, Motiani RK, Abdullaev IF, Bisaillon JM, Singer HA et al. Evidence for STIM1- and Orai1-dependent store-operated calcium influx through ICRAC in vascular smooth muscle cells: role in proliferation and migration. FASEB J 2009; 23: 2425-2437.

30. Smyth JT, Petranka JG, Boyles RR, DeHaven WI, Fukushima M, Johnson KL et al. Phosphorylation of STIM1 underlies suppression of store-operated calcium entry during mitosis. Nat Cell Biol 2009; 11: 1465-1472.

31. Lehen'kyi V, Flourakis M, Skryma R, Prevarskaya N. TRPV6 channel controls prostate cancer cell proliferation via $\mathrm{Ca}(2+) / \mathrm{NFAT}$-dependent pathways. Oncogene 2007; 26 : 7380-7385.

32. Livak KJ, Schmittgen TD. Analysis of relative gene expression data using real-time quantitative PCR and the 2(-Delta Delta $C(T))$ method. Methods 2001; 25: $402-408$.

Cell Death and Disease is an open-access journal published by Nature Publishing Group. This work is licensed under the Creative Commons Attribution-Noncommercial-No Derivative Works 3.0 Unported License. To view a copy of this license, visit http://creativecommons.org/licenses/by-nc-nd/3.0/ 